\title{
1,4-Dimethoxybutadienediyl-Bridged Diiron Compounds in Three Oxidation States: Evaluation of Delocalization Effects
}

\author{
Hiba Sahnoune,,$\star \|, \S$ Virginie Mahias, ${ }^{\S}$ Jean-François Halet, ${ }^{*} \S$ and Claude Lapinte ${ }^{*, \S}$ \\ *Département de Chimie, Faculté des Sciences, Université M'Hamed Bougara, 35000 Boumerdès, Algeria \\ "Laboratoire de Physique et Chimie Quantique, Faculté des Sciences, Université Mouloud Mammeri de Tizi- \\ Ouzou, 15000 Tizi-Ouzou, Algeria \\ §Univ Rennes, CNRS, ISCR-UMR 6226, F-350oo Rennes, France
}

\section{Supporting Information}

Including:

Page

1. Figure S1. ORTEP drawing of 1 meso $\left(\mathrm{PF}_{\mathbf{6}}\right)_{2} \quad$ S2

2. Figure S2. Experimental IR spectra (Nujol) 1meso $\left(\mathbf{P F}_{\mathbf{6}}\right)_{\mathbf{n}}(n=0-2) . \quad$ S3

3. Figure S3. ${ }^{57} \mathrm{Fe}$ Mössbauer spectra for 1 meso $\left(\mathbf{P F}_{6}\right)_{\mathbf{n}}$ at $80 \mathrm{~K}(n=0,1,2) \quad$ S4

4. Figure S4. ESR spectrum of 1 meso $\left(\mathbf{P F}_{6}\right)$ at $68 \mathrm{~K} \quad$ S5

5. Figure S5. ESR spectrum of $\mathbf{2}\left(\mathbf{P F}_{\mathbf{6}}\right)$ at $68 \mathrm{~K} \quad \mathrm{~S} 6$

6. Figure S6. ESR spectrum of $\mathbf{2}\left(\mathbf{P F}_{\mathbf{6}}\right)$ at $290 \mathrm{~K} \quad \mathrm{~S} 7$

7. Figure S7. Plots of the HOMO (-4.05 eV), HOMO-1 (-5.25 eV), LUMO $(0.26 \mathrm{eV})$, and $\mathrm{LUMO}+1(0.31 \mathrm{eV})$ of complex $1 \mathrm{dl}$. Contour values are $\pm 0.03\left(\mathrm{e} / \mathrm{bohr}^{3}\right)^{1 / 2}$

S8

8. Figure S8. Optimized geometrical $E$ and $Z$ configurations of $[1 \mathbf{m e s o}]^{2+} \quad$ S9

9. Figure S9. Hirshfeld atomic net charges for neutral 1meso (top) and cationic $[1 \text { meso }]^{+}$(bottom)

S10

10. Figure S10. Hirshfeld atomic net charges for neutral $\mathbf{1} \boldsymbol{d l}$ (top) and cationic $[\mathbf{1} \boldsymbol{d l}]^{+}$(bottom)

11. Figure S11. Hirshfeld atomic net charges for neutral 2 (top) and cationic $\mathbf{2}^{+}$ (bottom)

12. Figure S12. Hirshfeld atomic spin densities of cationic [1 meso $]^{+} \quad \mathrm{S} 13$

13. Figure S13. Hirshfeld spin densities of cationic [1 $d l^{+}{ }^{+} \quad S 14$

14. Figure S14. Hirshfeld atomic spin densities of cationic $\mathbf{2}^{+}$(top) and dicationic $\mathbf{2}^{2+}$ (bottom)

S15

15. Figure S15. Spatial distribution of the computed Mulliken spin density of $[\mathbf{1 d l}]^{+}$. Isocontour value: $\pm 0.002 \mathrm{e} / \mathrm{bohr}^{3}$. 
16. Table S1. Selected DFT-Optimized Bond Lengths $(\AA)$ and Angles $\left(^{\circ}\right)$ of $\mathbf{1}^{\mathbf{n}+}$ and $\mathbf{2}^{\mathbf{n}+}$ complexes $(n=0-2)$

17. Table S2. Computed Energy ( $\lambda, \mathrm{nm})$, Oscillator Strength $(f)$, Main Composition and Main Assignment of the First vis-NIR Electronic Excitations for Compounds $\mathbf{1}^{+}$and $\mathbf{2}^{+}$ 


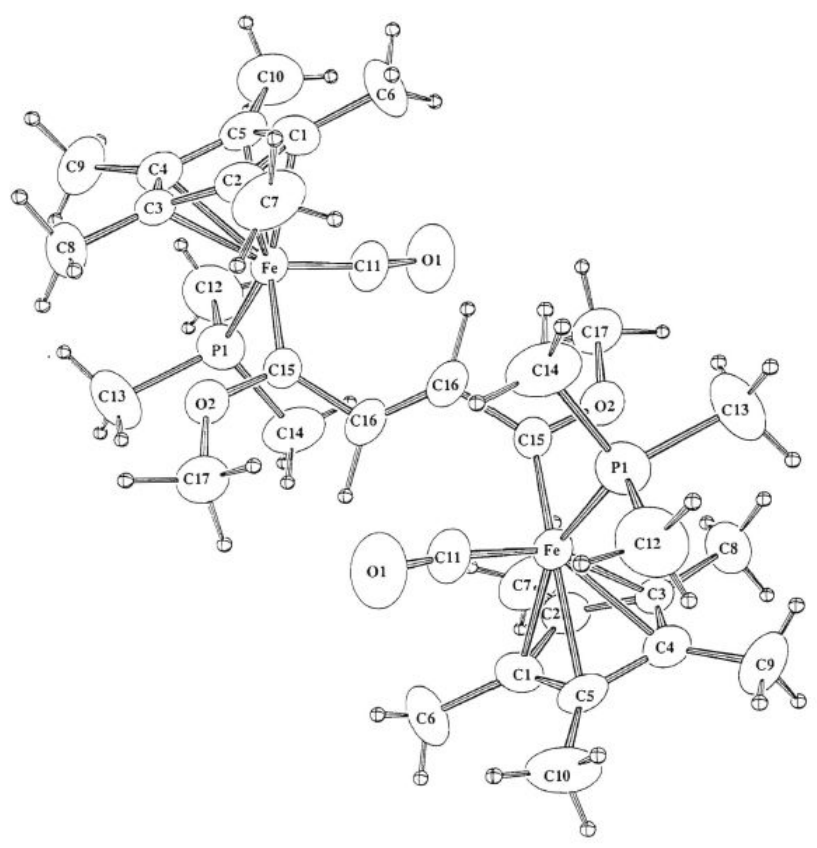

Figure S1. ORTEP drawing of $\mathbf{1}$ meso $\left(\mathbf{P F}_{\mathbf{6}}\right)_{2}$. Crystal system: orthorombic, space group $P b c a, Z=$ $4, a=10.887(2) \AA, b=16.667(2) \AA, c=24.018(3) \AA, R=0.052$ and $R_{\mathrm{w}}=0.049$. Selected bond lengths $(\AA)$ and angles $\left({ }^{\circ}\right)$ : Fe-P1 2.222(2), Fe-C11 1.743(8), Fe-C15 1.870(7), C15-C16 1.47(1), C16-C16 1.31(1), P1-Fe-C11 88.1(3), P1-Fe-C15 89.3(2), C11-Fe-C15 99.0(3), C15-C16-C16 127.0(9). 


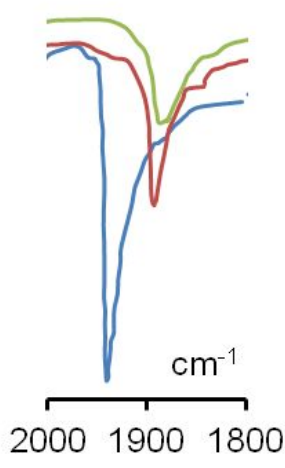

Figure S2. Experimental IR spectra (Nujol) $\mathbf{1 m e s o}\left(\mathbf{P F}_{\mathbf{6}}\right)_{\mathbf{n}}(n=0-2$, from top to bottom). 


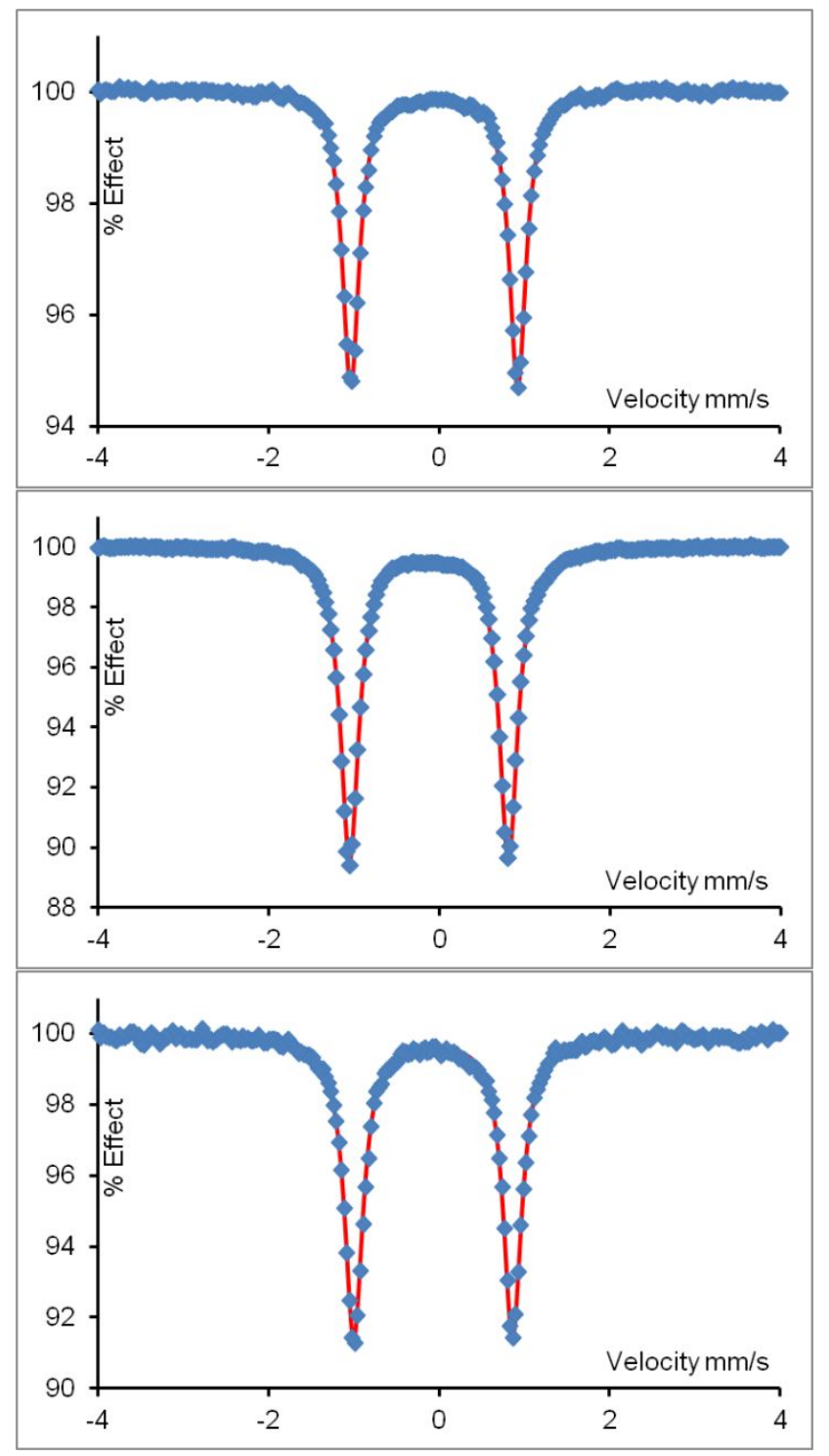

Figure S3. ${ }^{57} \mathrm{Fe}$ Mössbauer spectra for $\mathbf{1}$ meso $\left(\mathbf{P F}_{\mathbf{6}}\right)_{\mathbf{n}}$ at $80 \mathrm{~K}$ (from top to bottom, $n=0, n=1$, and $n=2$ ). 


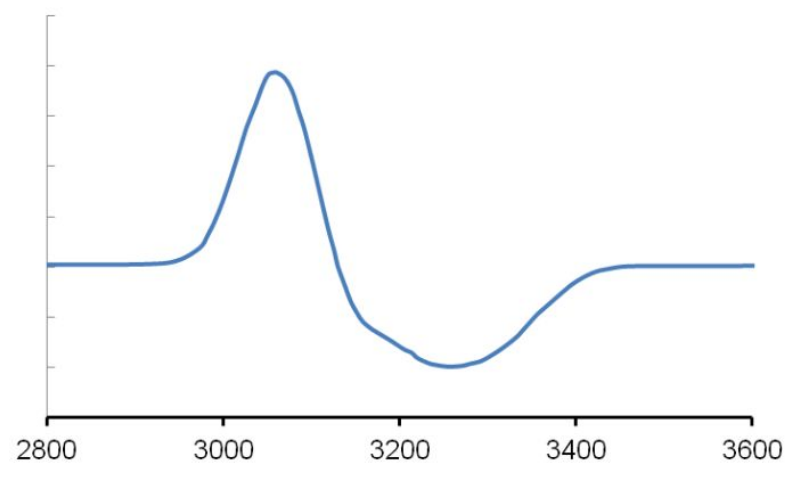

Figure S4. ESR spectrum of 1 meso $\left(\mathbf{P F}_{6}\right)$ in $1: 1 \mathrm{v} / \mathrm{v} \mathrm{CH}_{2} \mathrm{Cl}_{2} / \mathrm{C}_{2} \mathrm{H}_{4} \mathrm{Cl}_{2}$ at $68 \mathrm{~K}$ 


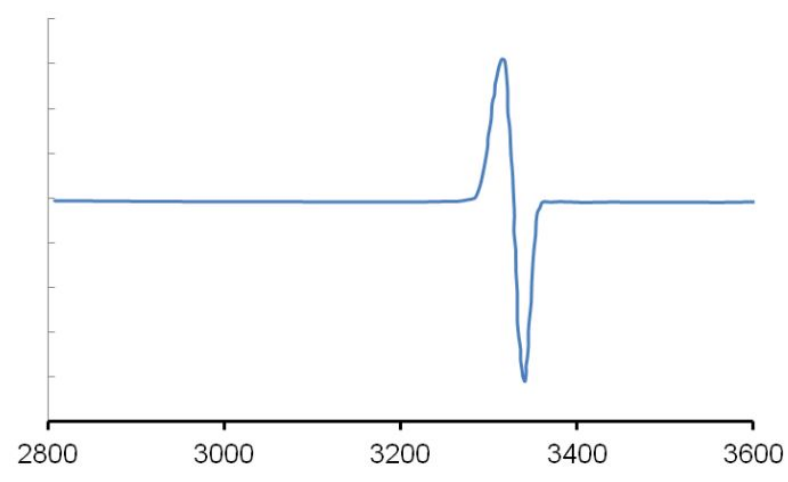

Figure S5. ESR spectrum of $\mathbf{2}\left(\mathbf{P F}_{\mathbf{6}}\right)$ in $1: 1 \mathrm{v} / \mathrm{v} \mathrm{CH}_{2} \mathrm{Cl}_{2} / \mathrm{C}_{2} \mathrm{H}_{4} \mathrm{Cl}_{2}$ at $68 \mathrm{~K}$ 


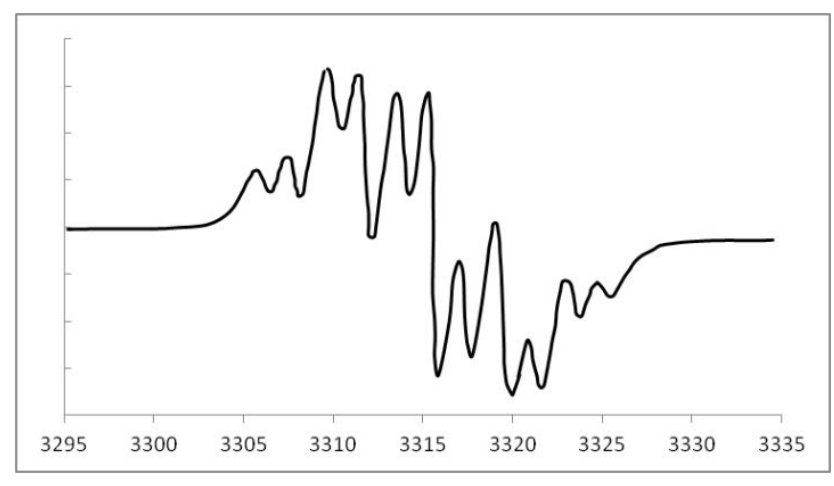

Figure S6. ESR spectrum of $\mathbf{2}\left(\mathbf{P F}_{\mathbf{6}}\right)$ in $1: 1 \mathrm{v} / \mathrm{v} \mathrm{CH}_{2} \mathrm{Cl}_{2} / \mathrm{C}_{2} \mathrm{H}_{4} \mathrm{Cl}_{2}$ at $290 \mathrm{~K}$ 


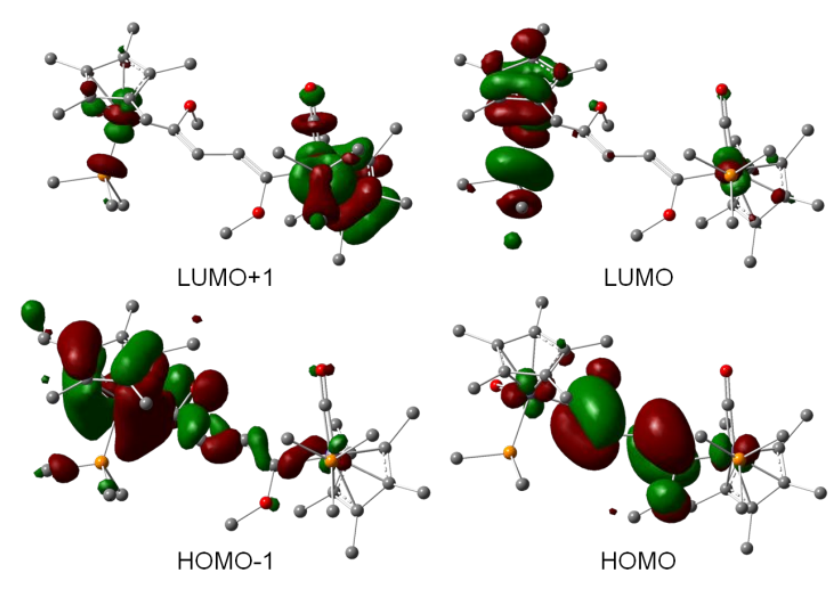

Figure S7. Plots of the HOMO (-4.05 eV), HOMO-1 (-5.25 eV), LUMO (0.26 eV), and LUMO+1 $(0.31 \mathrm{eV})$ of complex 1 dl. Contour values are $\pm 0.03\left(\mathrm{e} / \mathrm{bohr}^{3}\right)^{1 / 2}$. 


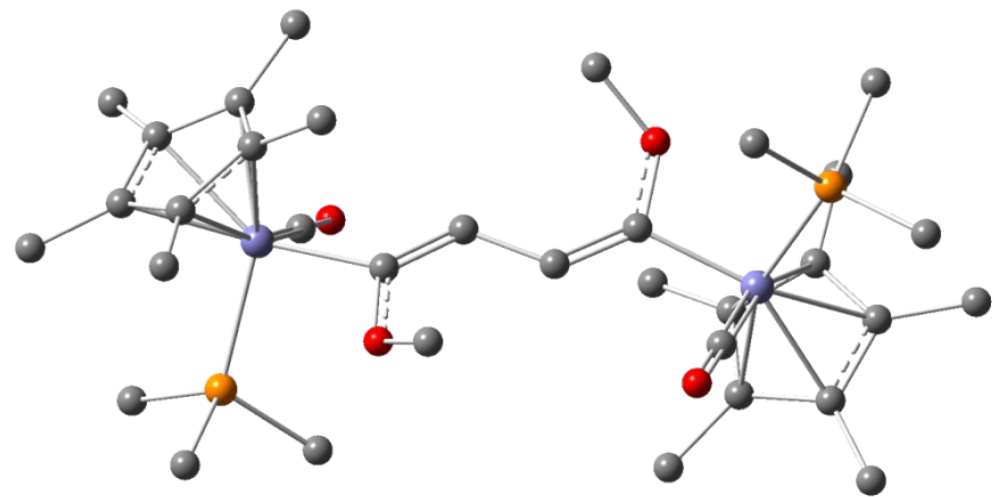

$(E)$

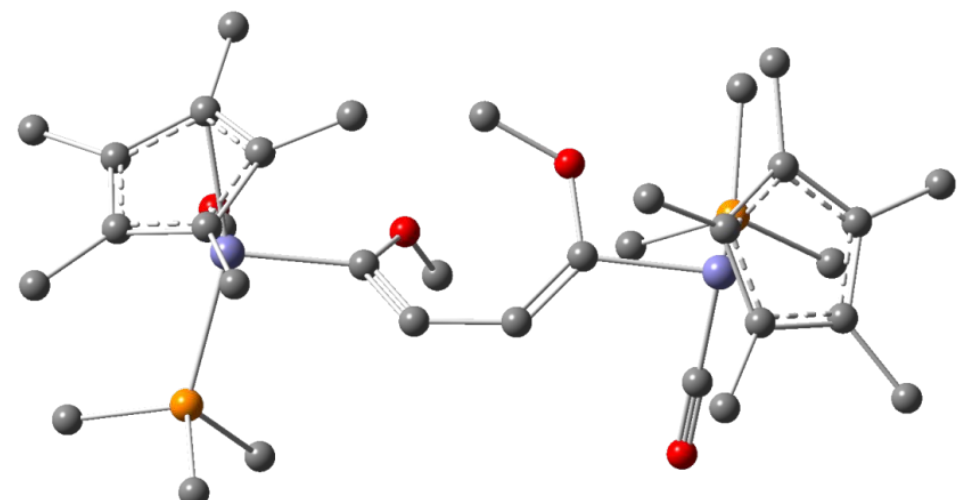

$(Z)$

Figure S8. Optimized geometrical $E$ and $Z$ configurations of $[1 \mathbf{m e s o}]^{2+}$. 

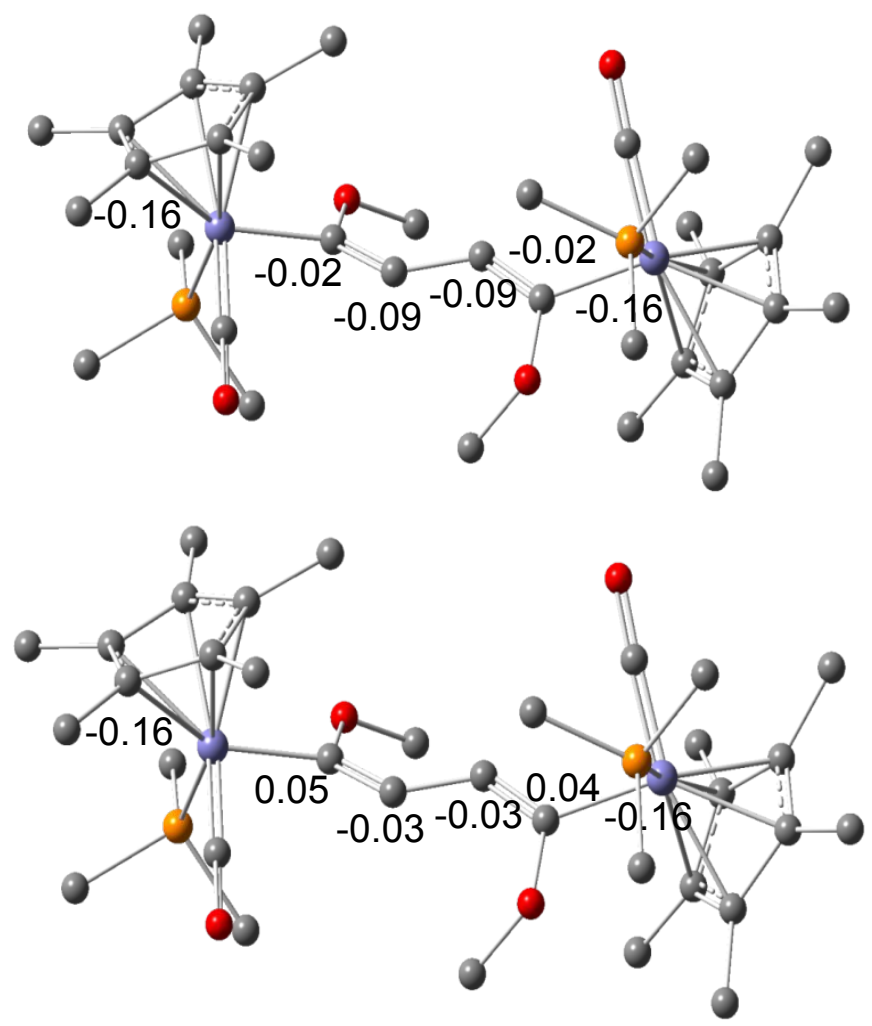

Figure S9. Hirshfeld atomic net charges for neutral 1 meso (top) and cationic $[\mathbf{1} \text { meso }]^{+}$(bottom). 

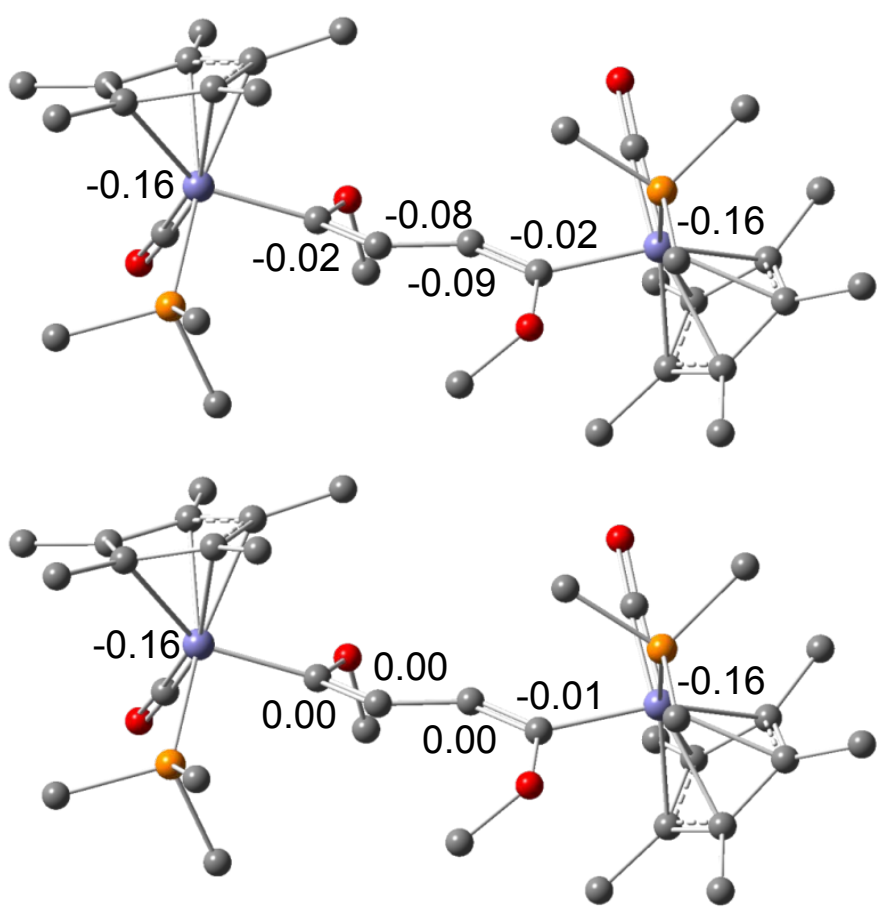

Figure S10. Hirshfeld atomic net charges for neutral $\mathbf{1} \boldsymbol{d} \boldsymbol{l}$ (top) and cationic $[\mathbf{1} \boldsymbol{d l}]^{+}$(bottom). 

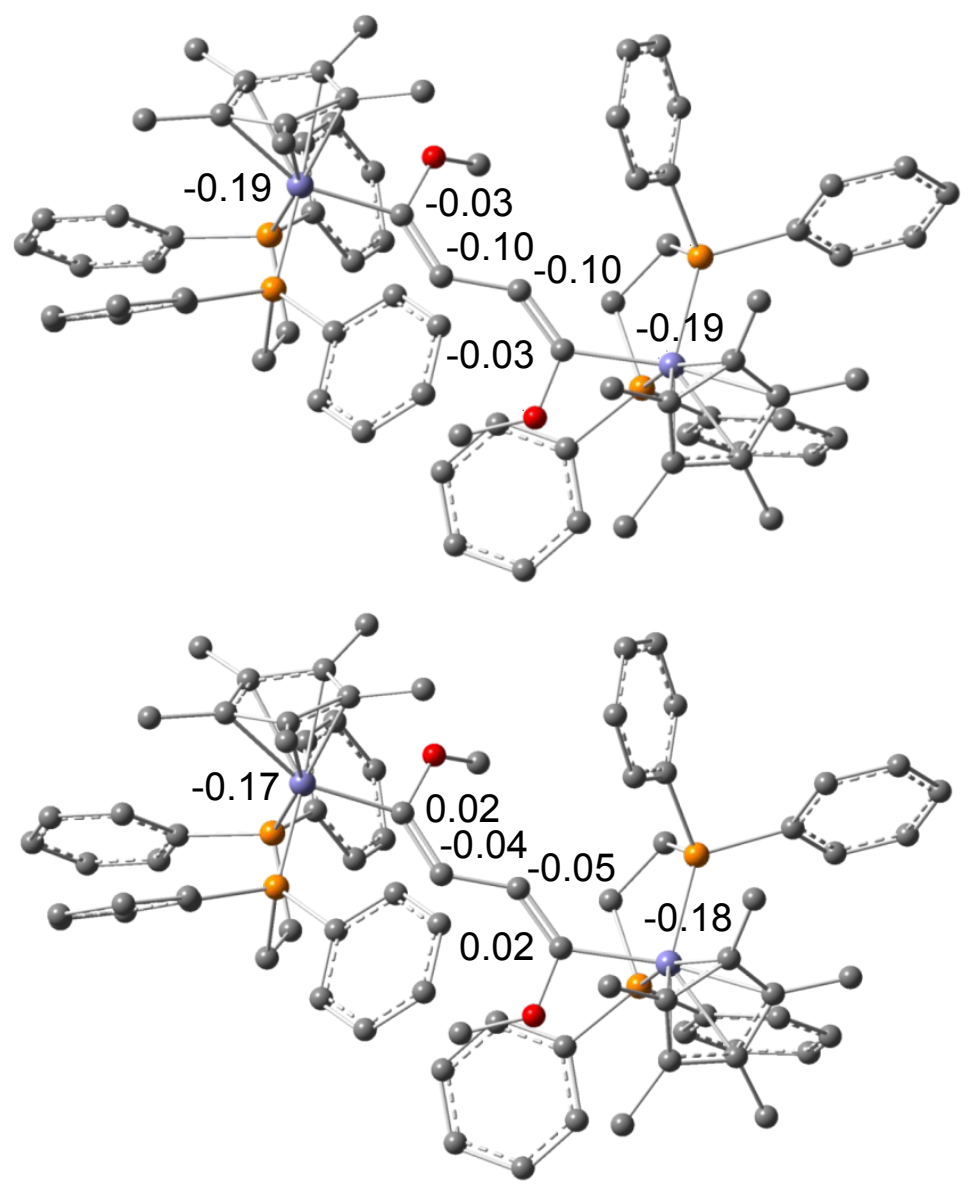

Figure S11. Hirshfeld atomic net charges for neutral 2 (top) and cationic $\mathbf{2}^{+}$(bottom). 


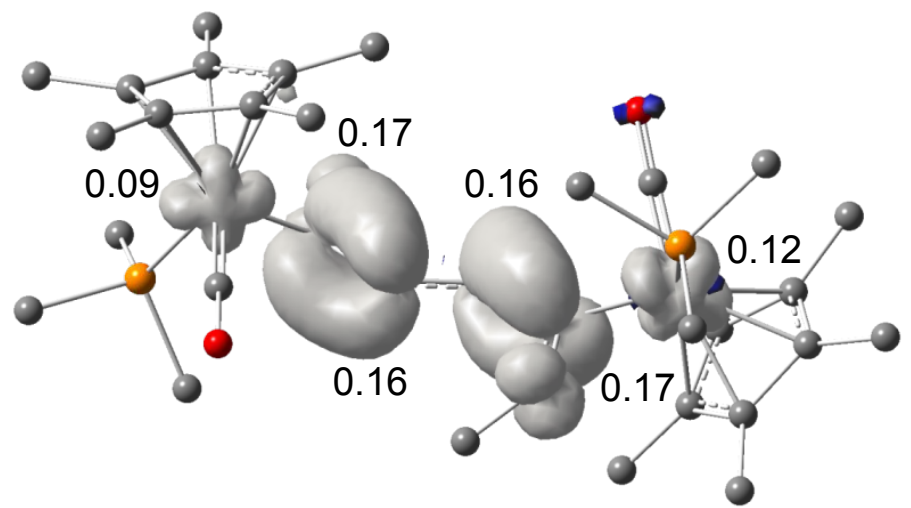

Figure S12. Hirshfeld atomic spin densities of cationic $[\mathbf{1} \text { meso }]^{+}$. 


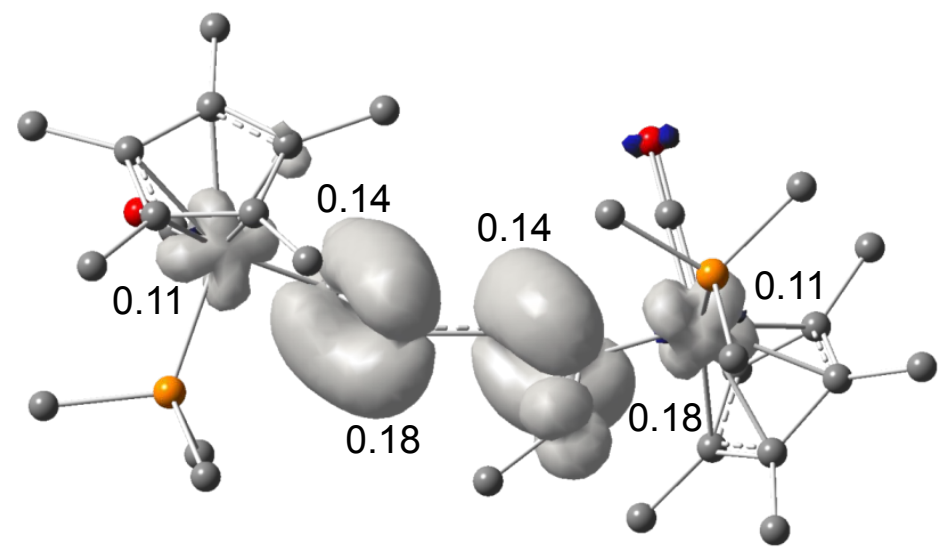

Figure S13. Hirshfeld spin densities of cationic $[\mathbf{1} \boldsymbol{d l}]^{+}$. 

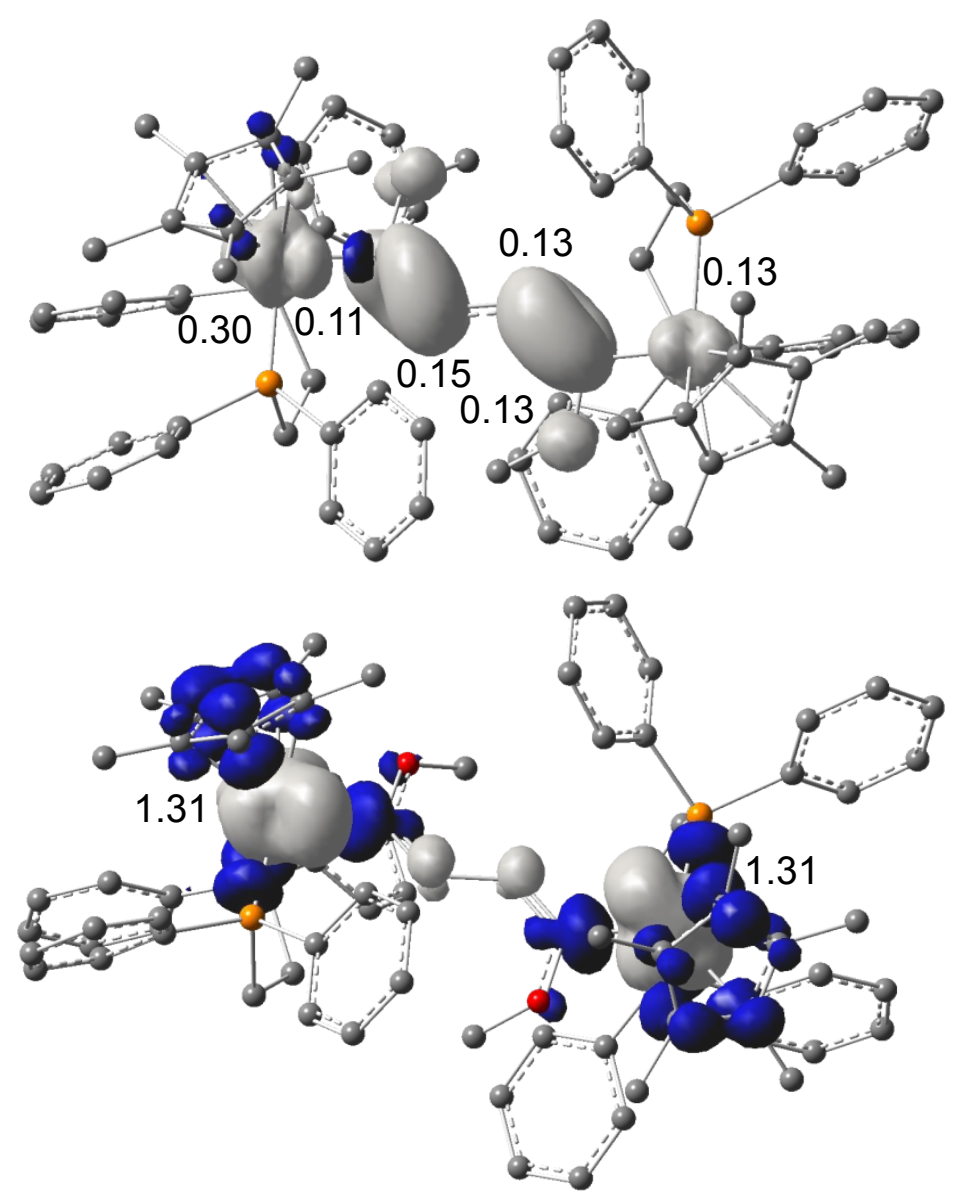

Figure S14. Hirshfeld atomic spin densities of cationic $\mathbf{2}^{+}$(top) and dicationic $\mathbf{2}^{\mathbf{2}}$ (bottom). 


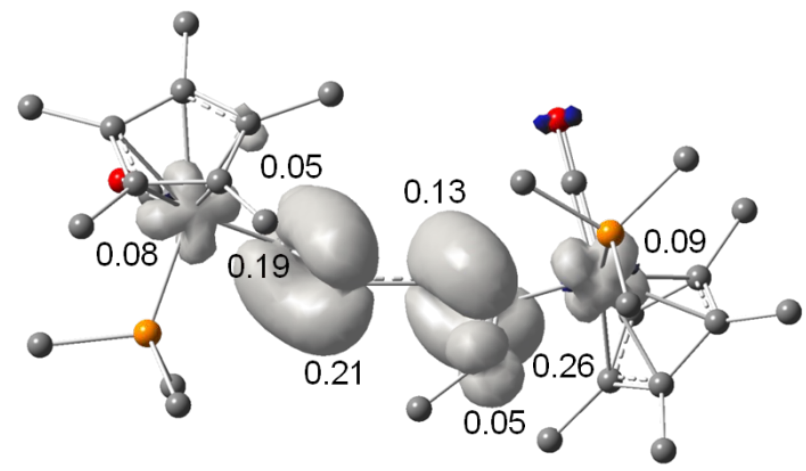

Figure S15. Spatial distribution of the computed Mulliken spin density of $[1 \mathbf{d l}]^{+}$. Isocontour value: \pm $0.002 \mathrm{e} / \mathrm{bohr}^{3}$. 
Table S1. Selected DFT-Optimized Bond Lengths $(\AA)$ and Angles $\left(^{\circ}\right)$ of $\mathbf{1}^{\mathbf{n}+}$ and $\mathbf{2}^{\mathbf{n}+}$ complexes $(n=$ 0-2)

\begin{tabular}{|c|c|c|c|c|c|c|c|c|c|c|c|c|}
\hline & 1 meso ${ }^{a}$ & {$[1 \mathrm{meso}]^{+\mathrm{a}}$} & $\begin{array}{l}{[\mathbf{1} \mathbf{m e s o}]^{2+}} \\
(\mathbf{S})^{a, b}\end{array}$ & $\begin{array}{l}{[1 \mathrm{meso} o]^{2+}} \\
(\mathrm{T})^{a, c}\end{array}$ & $1 d l$ & {$[1 d l]^{+}$} & $\begin{array}{l}{[1 d l]^{2+}} \\
(\mathbf{S})^{b}\end{array}$ & $\begin{array}{l}{[1 d l]^{2+}} \\
(\mathbf{T})^{c}\end{array}$ & 2 & {$[2]^{+}$} & $\begin{array}{l}{[2]^{2+}} \\
(\mathbf{S})^{c}\end{array}$ & $\begin{array}{l}{[2]^{2+}} \\
(\mathbf{T})^{c}\end{array}$ \\
\hline $\begin{array}{l}\mathrm{Fe} 1-\mathrm{Cp}^{*} \\
\text { (centroid) }\end{array}$ & 1.747 & 1.748 & 1.753 & 1.772 & 1.749 & 1.754 & 1.752 & 1.777 & 1.803 & 1.816 & 1.817 & 1.834 \\
\hline $\begin{array}{l}\mathrm{Fe} 2-\mathrm{Cp} * \\
\text { (centroid) }\end{array}$ & 1.746 & 1.747 & 1.760 & 1.772 & 1.745 & 1.749 & 1.762 & 1.768 & 1.797 & 1.805 & 1.826 & 1.834 \\
\hline Fe1-P1 & 2.195 & 2.216 & 2.236 & 2.322 & 2.195 & 2.208 & 2.235 & 2.311 & 2.215 & 2.246 & 2.239 & 2.358 \\
\hline Fe2-P2 & 2.200 & 2.217 & 2.231 & 2.324 & 2.199 & 2.218 & 2.239 & 2.323 & 2.197 & 2.226 & 2.247 & 2.287 \\
\hline $\begin{array}{l}\text { Fe1-P3 } \\
\text { Fe2-P4 }\end{array}$ & & & & & & & & & $\begin{array}{l}2.225 \\
2.205\end{array}$ & $\begin{array}{l}2.251 \\
2.231\end{array}$ & $\begin{array}{l}2.280 \\
2.268\end{array}$ & $\begin{array}{l}2.359 \\
2.287\end{array}$ \\
\hline $\mathrm{Fe} 1-\mathrm{C} 1$ & 1.958 & 1.913 & 1.840 & 1.967 & 1.956 & 1.907 & 1.837 & 1.965 & 2.031 & 1.947 & 1.896 & 2.027 \\
\hline $\begin{array}{l}\mathrm{C} 1-\mathrm{C} 2 \\
\mathrm{C} 2-\mathrm{C} 3\end{array}$ & $\begin{array}{l}1.362 \\
1.470\end{array}$ & $\begin{array}{l}1.417 \\
1.411\end{array}$ & $\begin{array}{l}1.479 \\
1.359\end{array}$ & $\begin{array}{l}1.358 \\
1.461\end{array}$ & $\begin{array}{l}1.361 \\
1.467\end{array}$ & $\begin{array}{l}1.421 \\
1.413\end{array}$ & $\begin{array}{l}1.475 \\
1.359\end{array}$ & $\begin{array}{l}1.364 \\
1.462\end{array}$ & $\begin{array}{l}1.372 \\
1.476\end{array}$ & $\begin{array}{l}1.420 \\
1.423\end{array}$ & $\begin{array}{l}1.486 \\
1.376\end{array}$ & $\begin{array}{l}1.370 \\
1.469\end{array}$ \\
\hline $\mathrm{C} 3-\mathrm{C} 4$ & 1.363 & 1.417 & 1.481 & 1.358 & 1.363 & 1.415 & 1.487 & 1.362 & 1.374 & 1.421 & 1.483 & 1.370 \\
\hline $\mathrm{Fe} 2-\mathrm{C} 4$ & 1.959 & 1.909 & 1.837 & 1.964 & 1.959 & 1.915 & 1.860 & 1.975 & 2.038 & 1.975 & 1.868 & 2.027 \\
\hline $\begin{array}{l}\mathrm{C} 1-\mathrm{O} 1 \\
\mathrm{O} 1-\mathrm{C} 5\end{array}$ & $\begin{array}{l}1.395 \\
1.416\end{array}$ & $\begin{array}{l}1.349 \\
1.428\end{array}$ & $\begin{array}{l}1.315 \\
1.444\end{array}$ & $\begin{array}{l}1.373 \\
1.433\end{array}$ & $\begin{array}{l}1.406 \\
1.410\end{array}$ & $\begin{array}{l}1.346 \\
1.427\end{array}$ & $\begin{array}{l}1.309 \\
1.444\end{array}$ & $\begin{array}{l}1.375 \\
1.423\end{array}$ & $\begin{array}{l}1.394 \\
1.409\end{array}$ & $\begin{array}{l}1.358 \\
1.420\end{array}$ & $\begin{array}{l}1.329 \\
1.424\end{array}$ & $\begin{array}{l}1.368 \\
1.417\end{array}$ \\
\hline $\mathrm{C} 4-\mathrm{O} 2$ & 1.397 & 1.349 & 1.319 & 1.373 & 1.398 & 1.353 & 1.316 & 1.365 & 1.385 & 1.350 & 1.334 & 1.367 \\
\hline $\mathrm{O} 2-\mathrm{C} 6$ & 1.419 & 1.432 & 1.441 & 1.433 & 1.414 & 1.427 & 1.432 & 1.425 & 1.407 & 1.417 & 1.429 & 1.417 \\
\hline $\begin{array}{l}\mathrm{Fe} 1-\mathrm{C} 7 \\
\mathrm{C} 7-\mathrm{O} 3\end{array}$ & $\begin{array}{l}1.707 \\
1.168\end{array}$ & $\begin{array}{l}1.716 \\
1.164\end{array}$ & $\begin{array}{l}1.731 \\
1.160\end{array}$ & $\begin{array}{l}1.748 \\
1.152\end{array}$ & $\begin{array}{l}1.709 \\
1.167\end{array}$ & $\begin{array}{l}1.729 \\
1.158\end{array}$ & $\begin{array}{l}1.741 \\
1.157\end{array}$ & $\begin{array}{l}1.754 \\
1.150\end{array}$ & & & & \\
\hline $\mathrm{Fe} 2-\mathrm{C} 8$ & 1.709 & 1.721 & 1.732 & 1.747 & 1.711 & 1.721 & 1.734 & 1.756 & & & & \\
\hline $\mathrm{C} 8-\mathrm{O} 4$ & 1.166 & 1.163 & 1.159 & 1.151 & 1.165 & 1.162 & 1.157 & 1.150 & & & & \\
\hline P1-Fe1-C1 & 88.6 & 89.7 & 92.3 & 97.4 & 92.2 & 88.4 & 92.4 & 98.4 & 94.5 & 97.4 & 86.6 & 101.8 \\
\hline $\mathrm{P} 2-\mathrm{Fe} 2-\mathrm{C} 4$ & 86.5 & 89.7 & 89.3 & 95.6 & 87.0 & 90.4 & 92.6 & 95.2 & 96.8 & 98.9 & 85.4 & 101.9 \\
\hline $\mathrm{P} 1-\mathrm{Fe} 1-\mathrm{C} 7$ & 89.4 & 89.9 & 90.4 & 86.4 & 88.7 & 89.1 & 90.5 & 87.7 & & & & \\
\hline $\mathrm{P} 2-\mathrm{Fe} 2-\mathrm{C} 8$ & 89.4 & 89.8 & 90.0 & 86.6 & 89.2 & 89.8 & 86.6 & 86.3 & & & & \\
\hline $\mathrm{C} 1-\mathrm{Fe} 1-\mathrm{C} 7$ & 91.1 & 94.1 & 96.3 & 84.7 & 92.1 & 92.6 & 97.0 & 89.2 & & & & \\
\hline $\mathrm{C} 4-\mathrm{Fe} 2-\mathrm{C} 8$ & 91.4 & 93.9 & 97.9 & 84.7 & 91.5 & 93.5 & 100.2 & 86.7 & & & & \\
\hline $\mathrm{Fe} 1-\mathrm{C} 1-\mathrm{C} 2$ & 125.4 & 123.5 & 120.8 & 129.2 & 128.9 & 123.8 & 117.2 & 121.5 & 132.8 & 131.4 & 131.2 & 128.4 \\
\hline $\mathrm{Fe} 2-\mathrm{C} 4-\mathrm{C} 3$ & 127.0 & 123.6 & 121.7 & 131.3 & 126.4 & 123.6 & 121.2 & 128.4 & 132.9 & 131.9 & 130.2 & 128.4 \\
\hline $\mathrm{C} 1-\mathrm{C} 2-\mathrm{C} 3$ & 130.7 & 130.5 & 126.3 & 130.0 & 124.5 & 128.1 & 128.0 & 123.0 & 129.9 & 129.3 & 132.7 & 129.8 \\
\hline $\mathrm{C} 2-\mathrm{C} 3-\mathrm{C} 4$ & 128.9 & 128.9 & 125.9 & 126.0 & 132.4 & 131.3 & 122.8 & 133.0 & 134.1 & 133.3 & 126.6 & 130.0 \\
\hline $\mathrm{P} 1-\mathrm{Fe} 1-\mathrm{P} 3$ & & & & & & & & & 85.8 & 85.5 & 85.5 & 83.4 \\
\hline P2-Fe2-P4 & & & & & & & & & 85.8 & 85.6 & 85.3 & 83.4 \\
\hline
\end{tabular}


Table S2. Computed Energy ( $\lambda, \mathrm{nm})$, Oscillator Strength $(f)$, Main Composition and Main Assignment of the First vis-NIR Electronic Excitations for Compounds $\mathbf{1}^{+}$and $\mathbf{2}^{+}$

\begin{tabular}{|c|c|c|c|c|}
\hline Compound & $\lambda \exp \mathrm{cm}^{-1}(\mathrm{~nm})$ & $\lambda$ calc nm $(f)$ & Composition & Main assignement \\
\hline \multirow[t]{6}{*}{$1^{+}$meso } & $\begin{array}{l}14720(680) \\
\text { D: } \text { LMCT }^{a}\end{array}$ & $686(0.04)$ & $36 \% \mathrm{HOSO} \beta \rightarrow \mathrm{LUSO}$ & $\mathrm{FeCp}^{*} \rightarrow \mathrm{CH}_{3} \mathrm{OC}=\mathrm{C}-\mathrm{C}=\mathrm{C}-\mathrm{OCH}_{3}(\mathrm{MLCT}+\mathrm{LLCT})$ \\
\hline & & & $21 \% \mathrm{HOSO} \beta-1 \rightarrow$ LUSO & $\mathrm{FeCp}^{*} \rightarrow \mathrm{CH}_{3} \mathrm{OC}=\mathrm{C}-\mathrm{C}=\mathrm{C}-\mathrm{OCH}_{3}$ \\
\hline & & $667(0.0004)$ & $\begin{array}{l}18 \% \mathrm{HOSO} \beta \rightarrow \text { LUSO }+2 \\
17 \% \mathrm{HOSO} \alpha-1 \rightarrow \text { LUSO }+2\end{array}$ & $\mathrm{FeCp}^{*} \rightarrow \mathrm{CpP}\left(\mathrm{CH}_{3}\right)_{3} \mathrm{CO}(\mathrm{MLCT}+\mathrm{LLCT})$ \\
\hline & & & & $\mathrm{FeCp}^{*} \rightarrow \mathrm{CpP}\left(\mathrm{CH}_{3}\right)_{3} \mathrm{CO}$ \\
\hline & & & $52 \%$ HOSO $\beta \rightarrow$ LUSO & $\mathrm{FeCp}^{*} \rightarrow \mathrm{CH}_{3} \mathrm{OC}=\mathrm{C}-\mathrm{C}=\mathrm{C}-\mathrm{OCH}_{3}(\mathrm{MLCT}+\mathrm{LLCT})$ \\
\hline & & & & $\mathrm{FeCp}^{*} \rightarrow \mathrm{CH}_{3} \mathrm{OC}=\mathrm{C}-\mathrm{C}=\mathrm{C}-\mathrm{OCH}_{3}$ \\
\hline \multirow[t]{6}{*}{$1^{+} d l$} & & $699(0.04)$ & $56 \% \mathrm{HOSO} \beta \rightarrow \mathrm{LUSO}$ & $\mathrm{FeCp}^{*} \rightarrow \mathrm{CH}_{3} \mathrm{OC}=\mathrm{C}-\mathrm{C}=\mathrm{C}-\mathrm{OCH}_{3}(\mathrm{MLCT}+\mathrm{LLCT})$ \\
\hline & & $676(0.004)$ & $43 \%$ HOSO $\beta-1 \rightarrow$ LUSO & $\mathrm{FeCp}^{*} \rightarrow \mathrm{CH}_{3} \mathrm{OC}=\mathrm{C}-\mathrm{C}=\mathrm{C}-\mathrm{OCH}_{3}(\mathrm{MLCT}+\mathrm{LLCT})$ \\
\hline & & $621(0.02)$ & $23 \% \mathrm{HOSO} \beta \rightarrow \mathrm{LUSO}$ & $\mathrm{FeCp}^{*} \rightarrow \mathrm{CH}_{3} \mathrm{OC}=\mathrm{C}-\mathrm{C}=\mathrm{C}-\mathrm{OCH}_{3}(\mathrm{MLCT}+\mathrm{LLCT})$ \\
\hline & & & $18 \%$ HOSO $\beta-1 \rightarrow$ LUSO & $\begin{array}{l}\mathrm{FeCp}^{*} \rightarrow \mathrm{CH}_{3} \mathrm{OC}=\mathrm{C}-\mathrm{C}=\mathrm{C}-\mathrm{OCH}_{3} \\
\mathrm{FeCp}^{*} \rightarrow \mathrm{Cp}^{*} \mathrm{P}\left(\mathrm{CH}_{3}\right)_{3} \mathrm{CO}(\mathrm{MLCT})\end{array}$ \\
\hline & & & $11 \% \mathrm{HOSO} \alpha-1 \rightarrow \mathrm{LUSO}+2$ & $\mathrm{FeCp}^{*} \rightarrow \mathrm{Cp}^{*} \mathrm{P}\left(\mathrm{CH}_{3}\right)_{3} \mathrm{CO}$ \\
\hline & & & $10 \% \mathrm{HOSO} \beta \rightarrow \mathrm{LUSO}+2$ & \\
\hline \multirow[t]{14}{*}{$\mathbf{2}^{+}$} & $8130(1230)$ & $1446(0.15)$ & $86 \% \mathrm{HOSO} \beta \rightarrow$ LUSO & $\mathrm{FeCH}_{3} \mathrm{OC}=\mathrm{C}-\mathrm{C}=\mathrm{C}-\mathrm{OCH}_{3} \mathrm{Fe}$ \\
\hline & A: $M->M$ & & & $(\mathrm{MLCT}+\mathrm{MMCT})$ \\
\hline & & 1254 & $5 \%$ HOSO $\beta-2 \rightarrow$ LUSO +18 & $\mathrm{FeCp}^{*} \rightarrow \mathrm{FeCp}^{*}(\mathrm{dppe})(\mathrm{MLCT}+\mathrm{MMCT})$ \\
\hline & & & $5 \%$ HOSO $\alpha-1 \rightarrow$ LUSO +18 & $\mathrm{FeCp}^{*} \rightarrow \mathrm{FeCp}^{*}(\mathrm{dppe})$ \\
\hline & $9870(1014)$ & 1067 & $9 \%$ HOSO $\beta-1 \rightarrow$ LUSO +18 & $\mathrm{FeCp}^{*} \rightarrow \mathrm{FeCp}^{*}(\mathrm{dppe})(\mathrm{MLCT}+\mathrm{MMCT})$ \\
\hline & B: $\mathrm{M}->\mathrm{M}$ & (0.0001) & $7 \%$ HOSO $\alpha-2 \rightarrow$ LUSO +18 & $\mathrm{FeCp}^{*} \rightarrow \mathrm{FeCp}^{*}(\mathrm{dppe})$ \\
\hline & & & $6 \% \mathrm{HOSO} \alpha-1 \rightarrow \mathrm{LUSO}+2 \mathrm{O}$ & $\mathrm{FeCp}^{*} \rightarrow \mathrm{FeCp}^{*}(\mathrm{dppe})$ \\
\hline & $11900(840)$ & $830(0.03)$ & $65 \%$ HOSO $\beta-1 \rightarrow$ LUSO & $\mathrm{FeCH}_{3} \mathrm{OC}=\mathrm{C}-\mathrm{C}=\mathrm{C}-\mathrm{OCH}_{3} \mathrm{Fe}$ \\
\hline & $\mathrm{C}: \mathrm{M}->\mathrm{M}$ & & & $(\mathrm{MLCT}+\mathrm{MMCT})$ \\
\hline & $16400(610)$ & $620(0.01)$ & $25 \%$ HOSO $\beta-5 \rightarrow$ LUSO & $\mathrm{FeCp}^{*} \rightarrow \mathrm{FeCH}_{3} \mathrm{OC}=\mathrm{C}-\mathrm{C}=\mathrm{C}-\mathrm{OCH}_{3} \mathrm{Fe}(\mathrm{MLCT}+\mathrm{MMCT})$ \\
\hline & D: MLCT & & $16 \%$ HOSO $\beta-8 \rightarrow$ LUSO & $\mathrm{FeCp}^{*} \rightarrow \mathrm{FeCH}_{3} \mathrm{OC}=\mathrm{C}-\mathrm{C}=\mathrm{C}-\mathrm{OCH}_{3} \mathrm{Fe}$ \\
\hline & & & $12 \% \mathrm{HOSO} \beta-4 \rightarrow$ LUSO & $\mathrm{FeCp}^{*} \rightarrow \mathrm{FeCH}_{3} \mathrm{OC}=\mathrm{C}-\mathrm{C}=\mathrm{C}-\mathrm{OCH}_{3} \mathrm{Fe}$ \\
\hline & $18720(534)$ & $536(0.02)$ & $40 \% \mathrm{HOSO} \beta-4 \rightarrow$ LUSO & $\mathrm{FeCp}^{*} \rightarrow \mathrm{FeCH}_{3} \mathrm{OC}=\mathrm{C}-\mathrm{C}=\mathrm{C}-\mathrm{OCH}_{3} \mathrm{Fe}(\mathrm{MLCT}+\mathrm{MMCT})$ \\
\hline & E: LMCT & & & \\
\hline
\end{tabular}

${ }^{a}$ See text and Table 5 . 